\title{
Bülent Karasözen
}

\section{Model order reduction in neuroscience}

\begin{abstract}
The human brain contains approximately $10^{9}$ neurons, each with approximately $10^{3}$ connections, synapses, with other neurons. Most sensory, cognitive, and motor functions of our brains depend on the interaction of a large population of neurons. In recent years, many technologies have been developed for recording large numbers of neurons either sequentially or simultaneously. Increases in computational power and algorithmic developments have enabled advanced analyses of the neuronal population parallel to the rapid growth of quantity and complexity of the recorded neuronal activity. Recent studies made use of dimensionality and model order reduction techniques to extract coherent features which are not apparent at the level of individual neurons. It has been observed that the neuronal activity evolves on low-dimensional subspaces. The aim of model reduction of large-scale neuronal networks is the accurate and fast prediction of patterns and their propagation in different areas of the brain. Spatiotemporal features of brain activity are identified on low-dimensional subspaces with methods such as dynamic mode decomposition, proper orthogonal decomposition, the discrete empirical interpolation method, and combined parameter and state reduction. In this chapter, we give an overview of the currently used dimensionality reduction and model order reduction techniques in neuroscience.
\end{abstract}

Keywords: neuroscience, dimensionality reduction, proper orthogonal decomposition, discrete empirical interpolation, dynamic mode decomposition, state and parameter estimation

MSC 2010: 93A15, 92C55, 37M10, 37M99, 37N40, 65R32

\subsection{Introduction}

Due to the advances in recording and imaging technologies, the number of recorded signals from brain cells increased significantly in the last few years. The recorded spatio-temporal neural activity gives rise to networks with complex dynamics. In neuroscience, molecular and cellular level details are incorporated in large-scale models of the brain in order to reproduce phenomena such as learning and behavior. The rapid growth of simultaneous neuronal recordings in scale and resolution brings challenges with the analysis of the neuronal population activity. New computational approaches have to be developed to analyze, visualize, and understand large-scale recordings of neural activity. While algorithmic developments and the availability of significantly

Bülent Karasözen, Department of Mathematics \& Institute of Applied Mathematics, Middle East Technical university, Ankara, Turkey

๑ Open Access. (C) 2021 Bülent Karasözen, published by De Gruyter. (cc) BY-NC-ND This work is licensed under the Creative Commons Attribution-NonCommercial-NoDerivatives 4.0 International License. 
more computing power have enabled analysis of larger neuronal networks, these advances cannot keep pace with the increasing size and complexity of recorded activity. The activity of complex networks of neurons can often be described by relatively few distinct patterns. Model order reduction techniques enable us to identify the coherent spatio-temporal patterns.

The presence or absence of a neural mechanism can be analyzed for neuronal populations. Dimensionality reduction methods [6] are data-driven statistical techniques for forming and evaluating hypotheses about population activity structure, which are summarized in Section 7.2. One of the goals of neuroscience is the fast and accurate prediction of the potential propagation in neurons. The differential equations describing the propagation of potential in neurons have been developed and are described by Hodgkin and Huxley equations [12]. They consist of a coupled system of ordinary and partial differential equations (ODEs and PDEs). The dimension of the associated discretized systems is very large for accurately simulating neurons with realistic morphological structure and synaptic inputs. In Section 7.3 we present two model order reduction approaches based on proper orthogonal decomposition (POD) and the discrete empirical interpolation method (DEIM) [5], which can predict accurately the potential propagation in large-scale neuronal networks leading to important speedups $[17,16,2]$. Using the functional neuroimaging data from electroencephalography (EEG) or functional magnetic resonance imaging (fMRI), different regions of the brain can be inferred by dynamic causal modeling (DCM) [7]. Effective connectivity is parameterized in terms of coupling among unobserved brain states and neuronal activity in different regions. In Section 7.4 we describe a combined state and parameter reduction for parameter estimation and identification [10] to extract effective connectivity in neuronal networks from measured data, such as EEG or fMRI data. In Section 7.5 the data-driven, equation-free model order reduction method dynamic mode decomposition (DMD) is described for identifying sleep spindle networks [3]. Reduced-order models (ROMs) with POD and the DEIM and four variants of them are presented for neuronal synaptic plasticity and neuronal spiking networks in Section 7.6.

\subsection{Dimensionality reduction methods}

Coordination of responses across neurons exists only at the level of the population and not at the level of single neurons. The presence or absence of a neural mechanism can be analyzed for neuronal populations. Dimensionality reduction methods are data-driven statistical techniques for forming and evaluating hypotheses about population activity structure. They produce low-dimensional representations of highdimensional data with the aim to extract coherent patterns which preserve or highlight some feature of interest in the data [6]. The recorded neurons of dimension $D$ are likely 
not independent of each other, because they belong to a common network of neuronal populations. From the high-dimensional data of neuronal recordings, a smaller number of explanatory variables $K(K<D)$ are extracted with the help of dimensionality reduction methods. The explanatory variables are not directly observed; therefore they are referred to as latent variables. The latent variables define a $K$-dimensional space representing coherent patterns of the noisy neural activity of $D$ neurons.

There exist several dimensionality reduction methods which differ in the statistical interpretation of the preserved and discarded features of the neuronal populations. We summarize the commonly used statistical methods of dimensionality reduction methods in [6], where further references about the methods can be found.

Principal component and factor analysis. The most widely known technique to extract coherent patterns from high-dimensional data is modal decomposition. A particularly popular modal decomposition technique is principal component analysis (PCA), which derives modes ordered by their ability to account for energy or variance in the data. In particular, PCA is a static technique and does not model temporal dynamics of time-series data explicitly, so it often performs poorly in reproducing dynamic data, such as recordings of neural activity. The low-dimensional space identified by PCA captures variance of all types, including firing rate variability and spiking variability, whereas factor analysis discards the independent variance for each neuron. and preserves variance that is shared across neurons.

Time series methods. The temporal dynamics of the population activity can be identified if the data come from a time series. The most commonly used time series methods for dimensionality reduction neural recordings are hidden Markov models (HMMs) [18], kernel smoothing followed by a static dimensionality reduction, Gaussian process factor analysis [35], latent linear dynamical systems [4], and latent nonlinear dynamical systems [26]. They produce latent neural trajectories that capture the shared variability across neurons. The HMM is applied when a jump between discrete states of neurons exists, other methods identify smooth changes in firing rates over time.

Methods with dependent variables. On many neuronal recordings the high-dimensional firing rate space is associated with labels of one or more dependent variables, like stimulus identity, decision identity, or a time index. The dimensionality reduction aims in this case to project the data such that differences in these dependent variables are preserved. Linear discriminant analysis can be used to find a low-dimensional projection in which the $G$ groups to which the data points belong are well separated.

Nonlinear dimensionality reduction methods. All the previous methods assume a linear relationship between the latent and observed variables. When the data lie on a low-dimensional, nonlinear manifold in the high-dimensional space, a linear method may require more latent variables than the number of true dimensions of the data. The most frequently used methods to identify nonlinear manifolds are Isomap79 [31] and locally linear embedding [28]. Because the nonlinear methods use local neighborhoods to estimate the structure of the manifold, population responses may not evenly 
explore the high-dimensional space. Therefore theses methods should be used with care.

\subsection{Proper orthogonal decomposition and discrete empirical interpolation for the Hodgkin-Huxley model}

One of the goals of neuroscience is the fast and accurate prediction of the potential propagation in neurons. The differential equations describing propagation of potential in neurons are described by Hodgkin-Huxley (HH) cable equations [12]. They consist of a coupled system of ODEs and PDEs. Accurate simulation of morphology, kinetics, and synaptic inputs of neurons requires solution of large systems of nonlinear ODEs. The complexity of the models is determined by the synapse density and the dentritic length. In simulations, for one synapse per micron on a cell with a dendrite of $5 \mathrm{~mm}, 5,000$ compartments each with 10 variables are needed, which results in 50,000 coupled nonlinear system of ODEs $[17,16]$. To recover complex dynamics, efficient reduced-order neuronal methods are developed using POD and the DEIM from the snapshots of the in space and time discretized coupled PDEs and ODEs [17, 16, 2]. In this section we describe two of them. They differ in the formulation of the $\mathrm{HH}$ cable equation and of the equations for the gating variables.

\subsubsection{Morphologically accurate reduced-order modeling}

The neuronal full-order models (FOMs) in $[17,16]$ consist of $D$ branched dendritic neurons $B=\sum_{d=1}^{D} B_{d}$ meeting at the soma, where the $d$-th neuron has $B_{d}$ branches. It is assumed that the branch $b$ carries $C$ distinct ionic currents with associated densities and $G_{b c}(x)$ and reversal potentials $E_{c}, c=1, \ldots, C$. The kinetics of current $c$ on branch $b$ are governed by the $F_{c}$ gating variables, $w_{b c f}, f=1, \ldots, F_{c}$. When subjected to input at $S_{b}$ synapses, the nonlinear $\mathrm{HH}$ cable equation for the transmembrane potential $v_{b}(x, t)$ with the equation for the gating variables $w_{b c f}$ is given by (see [2], Figure 1, model network with three cables)

$$
\begin{aligned}
a_{b} C_{m} \frac{\partial v_{b}}{\partial t}= & \frac{1}{2 R_{i}} \frac{\partial}{x}\left(a_{b}^{2} \frac{\partial v_{b}}{\partial x}\right) \\
& -a_{b} \sum_{c=1}^{C} G_{b c}(x)\left(v_{b}-E_{c}\right) \prod_{f=1}^{F_{c}} w_{b c f}^{q_{c f}} \\
& \frac{1}{2 \pi} \sum_{s=1}^{S_{b}} g_{b s}(t) \delta\left(x-x_{b s}\right)\left(v_{b}-E_{b s}\right),
\end{aligned}
$$




$$
\frac{\partial w_{b c f}}{\partial t}=\frac{w_{c f, \infty}\left(v_{b}\right)-w_{b c f}}{\tau_{c f}\left(v_{b}\right)}, \quad 0<x<l_{b}, t>0,
$$

where $g_{b s}(n S)$ is the time course, $x_{b s}$ is the spatial location, and $E_{b s}$ is the reversal potential of the $s$-th synapse on branch $b$. The variables and parameters in (7.1) are described in $[17,16]$.

These branch potentials interact at $J$ junction points, where junction $J$ denotes the soma. The $D$ dendrites join at the soma. Continuity of the potential at the soma leads to a common value at the current balance denoted by $v_{\sigma}(t)$. Then the networked form of (7.1) becomes

$$
\begin{aligned}
a_{b} C_{m} \frac{\partial v_{\sigma}}{\partial t}= & \frac{\pi}{A_{\sigma} R_{i}} \sum_{d=1}^{D} \frac{\partial}{\partial x}\left(a_{b_{J}^{d}}^{2}\left(l_{b_{J}^{d}}\right) \frac{\partial v_{b_{J^{d}}}\left(l_{b_{J^{d}}}, t\right)}{\partial x}\right) \\
& -a_{b} \sum_{c=1}^{C} G_{\sigma c}(x)\left(v_{\sigma}-E_{c}\right) \prod_{f=1}^{F_{c}} w_{\sigma c f}^{q_{c f}}(t) \\
& \frac{1}{A_{\sigma}} \sum_{s=1}^{S_{b}} g_{\sigma s}(t)\left(v_{\sigma}(t)-E_{\sigma S}\right), \\
\frac{\partial w_{\sigma c f}(t)}{\partial t}= & \frac{w_{c f, \infty}\left(v_{\sigma}(t)\right)-w_{\sigma c f}(t)}{\tau_{c f}\left(v_{\sigma}\right)(t)}, \quad 0<x<l_{b}, t>0 .
\end{aligned}
$$

When the cell is partitioned into $N$ compartments, with $C$ distinct ionic currents per compartment and with $F$ gating variables per current, the following nonlinear ODEs are obtained:

$$
\begin{aligned}
v^{\prime}(t)= & H v(t)-(\Phi(w(t)) e) \cdot v(t)+\Phi(w(t)) E_{i} \\
& +G(t) \cdot\left(v(t)-E_{S}\right), \quad v(t) \in \mathbb{R}^{N}, \\
w^{\prime}(t)= & (A(v(t))-w(t)) \cdot / B(v(t)), \quad w(t) \in \mathbb{R}^{N \times C \times F},
\end{aligned}
$$

where $H \in \mathbb{R}^{N \times N}$ is the Hines matrix [11], $e=\left[\begin{array}{lll}1 & 1 \cdots 1\end{array}\right]^{T} \in \mathbb{R}^{C}$ and the "dot" operator, $a . b$, denotes elementwise multiplication; $E_{i}$ and $E_{s}$ are the vector of channel reversal potentials and the vector of synaptic reversal potentials, respectively. Equation (7.5) is discretized in time by the second-order discretized Euler scheme [11].

In [16] using the snapshots of $v(t)$ and of the nonlinear term $N(v(t), w(t)) \equiv$ $(\Phi(w(t)) e) \cdot v(t)-\Phi(w(t)) E_{i}$ at times $t_{1}, t_{2}, \ldots, t_{n}$ the POD and DEIM modes are constructed.

The reduced membrane potentials $v_{r}$ are constructed using the POD basis, and the reduced gating variables $w_{r}$ are obtained after applying the DEIM to the nonlinear terms. The ROM in [16] preserves the spatial precision of synaptic input and captures accurately the subthreshold and spiking behaviors.

In [17] a linearized quasi-active reduced neuronal model is constructed using balanced truncation and $\mathcal{H}_{2}$-approximation of transfer functions in time. ROMs preserve the input-output relationship and reproduce only subthreshold dynamics. 


\subsubsection{Energy-stable neuronal reduced-order modeling}

In $[1,2]$ a different form of the HH cable equation and ODEs for gating variables is considered. The intracellular potential $v(x, t)$ and three gating variables $m(x, t), h(x, t)$, and $n(x, t)$ describe the activation and deactivation of the ion channels, of the sodium channels, and of the potassium channels, respectively. A single cable in the computational domain $(x, t) \in[0, L] \times(0, T]$ describing the distribution of the potential $u(x, t)$ is given by $[1,2]$

$$
\frac{\partial u}{\partial t}=\frac{\mu}{a(x)}\left(a(x)^{2} u_{x}\right)_{x}-\frac{1}{C_{m}} g(m, h, n) u+\frac{1}{C_{m}} f(m, h, n, x, t),
$$

where $\mu=\frac{1}{2 C_{m} R_{i}}>0, a(x)$ is the radius of the neurons, $C_{m}$ is the specific membrane capacitance, and $R_{i}$ is the axial resistivity. The conductance $g(x, t)$ is a polynomial of the gating variables

$$
g(x, t)=g_{1} m^{3} h+g_{2} n^{4}+g_{3}>0,
$$

with the source term

$$
f(m, h, n, x, t)=g_{1} E_{1} m^{2} h+g_{2} E_{2} n^{4}+g_{3} E_{3}-i(x, t),
$$

where $E_{l}, l=1,2,3$, are equilibrium potentials and $i(x, t)$ is the input current at $x$,

$$
i(x, t)=\sum_{s=1}^{N_{s}} i_{s}(x, t), \quad x \in[0, L] .
$$

The nonlinear ODEs for the gating variables are given by

$$
\begin{aligned}
& \frac{\partial m}{\partial t}=\alpha_{m}\left(v(x, t)(1-m(x, t))-\beta_{m} v(x, t)\right) m(x, t), \\
& \left.\frac{\partial h}{\partial t}=\alpha_{h}(v(x, t))(1-h(x, t))-\beta_{h} v(x, t)\right) h(x, t), \\
& \left.\frac{\partial n}{\partial t}=\alpha_{n}(v(x, t))(1-n(x, t))-\beta_{n} v(x, t)\right) n(x, t) .
\end{aligned}
$$

Expressions for $\alpha_{m}, \alpha_{h}, \alpha_{n}, \beta_{m}, \beta_{h}, \beta_{n}$ and boundary conditions can be found in [2].

In $[1,2]$, a model network with three cables connected to a soma is used. The equations governing the potential propagation in a network of $N_{c}$ neuron cables-dendrites and/or axons with the superscript ${ }^{(c)}, c=1, \ldots N_{c}$, are given as

$$
\begin{aligned}
\frac{\partial v^{(c)}}{\partial t}= & \frac{\mu}{a^{(c)}\left(\chi^{(c)}\right)}\left(\left(a^{(c)}\left(x^{(c)}\right)^{2}\right) v_{x}^{(c)}\right)_{x}-\frac{1}{C_{m}} g\left(m^{(c)}, h^{(c)}, n^{(c)}\right) u^{(c)} \\
& +\frac{1}{C_{m}} f\left(m^{(c)}, h^{(c)}, n^{(c)}, x^{(c)}, t\right),
\end{aligned}
$$




$$
\begin{aligned}
\frac{\partial m^{(c)}}{\partial t} & =\alpha_{m}\left(v^{(c)}\left(1-m^{(c)}\right)-\beta_{m} v^{(c)}\right) m^{(c)} \\
\frac{\partial h^{(c)}}{\partial t} & \left.=\alpha_{h}\left(v^{(c)}\right)\left(1-h^{(c)}\right)-\beta_{h} v(c)\right) h^{(c)} \\
\frac{\partial n^{(c)}}{\partial t} & \left.\left.=\alpha_{n}\left(v^{(c)}\right)\right)\left(1-n^{(c)}\right)-\beta_{n} v^{(c)}\right) n^{(c)}
\end{aligned}
$$

for $x^{(c)} \in \Omega^{(c)}=\left[0, L^{(c)}\right]$ together with the boundary conditions.

The semi-discrete forms of these equations are approximated using energy-stable summation by parts [1, 2] for the model network. The reduced-order bases (ROBs) for multiple cables of identical lengths are assembled into a network in block form. The block structure of the ROB allows a flexible structure-preserving model reduction approach with an independent approximation in each cable and energy stability and accuracy properties follow from this block structure. Computation of the time-varying reduced variables in the gating equations at every time $t$ is costly because they scale with the dimension of the FOM. A nonnegative variant of the DEIM, nonnegative DEIM (NNDEIM), is developed in [2] to preserve the structure and energy stability properties of the equations.

The capability of the greedy-based approach to generate accurate predictions in large-scale neuronal networks is demonstrated for systems with more than 15,000 degrees of freedom. The state variable ROB of dimension $l=15$ with POD modes and the nonnegative ROBs of dimension $p=60$ with NNDEIM modes are constructed using a greedy approach to predict the potential variation at the soma. The speedup factor of simulations is about 20, which is larger than that of Galerkin projection, which is only 1.3 without the NNDEIM.

\subsection{Combined state and parameter reduction for dynamic causal modeling}

In neuroscience, different regions of the brain are inferred using neuroimaging data from EEG or fMRI recordings using the method of DCM [7]. Effective connectivity is parameterized in terms of coupling among unobserved brain states and neuronal activity in different regions. In DCM the neuronal activity of the observed brain region is represented as a single-input single-output (SISO) system

$$
\dot{x}=A_{\mathrm{dyn}}(\mu) x+B_{\mathrm{dyn}} u,
$$

with the parameterized connectivity $A_{\mathrm{dyn}}(\mu)$ and external input matrices $B_{\mathrm{dyn}}$.

Linearization of the nonlinear DCM hemodynamic forward submodel (balloon model) [7] transforms the neuronal activity to the measured blood oxygen leveldependent (BOLD) response. Linearization around the equilibrium results in the 
following SISO system:

$$
\begin{aligned}
& B_{\mathrm{obs}}:=\left(\begin{array}{llll}
1 & 0 & 0 & 0
\end{array}\right)^{T}, \quad C_{\mathrm{obs}}=\left(\begin{array}{llll}
0 & 0 & V_{0} k_{1} & V_{0} k_{2}
\end{array}\right), \\
& \dot{z}_{i}=A_{\mathrm{obs}} z_{i}+B_{\mathrm{obs}} x_{i}, \\
& y_{i}=C_{\mathrm{obs}} z_{i}, \\
& z_{0}=\left(\begin{array}{llll}
0 & 0 & 0 & 0
\end{array}\right)^{T}, \\
& A_{\mathrm{obs}}:=\left(\begin{array}{cccc}
\frac{1}{\tau_{s}} & \frac{1}{\tau_{f}} & 0 & 0 \\
1 & 0 & 0 & 0 \\
0 & \frac{1}{\tau_{0} E_{0}}\left(\begin{array}{cccc}
\left.1-\left(1-E_{0}\right)\left(1-\ln \left(1-E_{0}\right)\right)\right) & \frac{1}{\tau_{0}} & \frac{1-\alpha}{\tau_{0} \alpha} \\
0 & \frac{1}{\tau_{0}} & 0 & \frac{1}{\tau_{0} \alpha}
\end{array}\right) .
\end{array}\right.
\end{aligned}
$$

The fMRI measurements at the $i$-th brain region are reflected by the output variables $y_{i}$. For the meaning of the variables and parameters in (7.15) and (7.19) we refer to $[10,9]$. The linearized forward submodels are embedded into the fMRI connectivity model

$$
\begin{aligned}
& \left(\begin{array}{c}
\dot{x} \\
\dot{z}_{1} \\
\dot{z}_{2} \\
\vdots \\
z_{N_{\mathrm{dyn}}}
\end{array}\right)=\left(\begin{array}{ccccc}
A_{\mathrm{dyn}}(\mu) & 0 & 0 & \cdots & 0 \\
\delta_{1,1} & A_{\mathrm{obs}} & 0 & & \\
\delta_{2,1} & 0 & A_{\mathrm{obs}} & & \\
\vdots & & \ddots & & \\
\delta_{1, N_{\mathrm{dyn}}} & & & A_{\mathrm{obs}} &
\end{array}\right)\left(\begin{array}{c}
x \\
z_{1} \\
z_{2} \\
\vdots \\
z_{N_{\mathrm{dyn}}}
\end{array}\right) \\
& +\left(\begin{array}{c}
B_{\mathrm{dyn}} \\
0 \\
0 \\
\vdots \\
0
\end{array}\right) v \\
& y=\left(0\left(\begin{array}{ccc}
C_{\mathrm{obs}} & & \\
& \ddots & \\
& & C_{\mathrm{obs}}
\end{array}\right)\right)\left(\begin{array}{c}
x \\
z_{1} \\
z_{2} \\
\vdots \\
z_{N_{\mathrm{dyn}}}
\end{array}\right),
\end{aligned}
$$

where $\delta_{i j} \in \mathbb{R}^{4 \times N_{\mathrm{dyn}}}$ denotes the Kronecker matrix with nonzero elements located at the $(i, j)$-th component.

The linearized state-space forward model (7.20) and (7.21) corresponds to a multiple-input multiple-output (MIMO) system,

$$
\dot{x}(t)=A(\mu) x(t)+B u(t), \quad y(t)=C x(t),
$$


where $x \in \mathbb{R}^{N}$ is the internal state, $u \in \mathbb{R}^{J}$ is the external input, $y \in \mathbb{R}^{O}$ is the observed output, and $\mu$ are the parameters describing different conditions.

For large numbers of $M:=N^{2}$ parameters, the computational cost for inferring the parameters and states is very high. In $[10,8]$ a combined state and parameter model order reduction is developed for parameter estimation and identification to extract effective connectivity. The inversion procedure consists of two phases, the offline and online phases. In the offline phase, the underlying parameterized model is reduced jointly in states and parameters. In the online phase, the ROM's parameters are estimated to fit the observed experimental data. Using state and parameter reduction, the computational cost is reduced in the offline phase. The simultaneous reduction of state and parameter space is based on Galerkin projections with the orthogonal matrices for the state $V \in \mathbb{R}^{N \times n}$ and for the parameters $P \in \mathbb{R}^{M \times m}$. The reduced model is of lower order $n \ll N, m \ll M$ than the original FOM. The reduced states $x_{r}(t) \in \mathbb{R}^{n}$ and the reduced parameters $\mu \in \mathbb{R}^{m}$ are computed as

$$
\dot{x}_{r}(t)=A_{r}\left(\mu_{r}\right) x_{r}(t)+B_{r} u(t), \quad y_{r}(t)=C_{r} x(t),
$$

with a reduced initial condition $x_{r, 0}=V^{T} x_{0}$ and the reduced components

$$
\begin{aligned}
\mu_{r} & =P^{T} \mu \in \mathbb{R}^{m}, \\
A_{r}\left(\mu_{r}\right) & =V^{T} A\left(P \mu_{r}\right) V \in \mathbb{R}^{n \times n}, \\
B_{r} & =V^{T} B \in \mathbb{R}^{n \times J}, \\
C_{r} & =C V \in \mathbb{R}^{O \times m} .
\end{aligned}
$$

In the online phase, the optimization-based inverse problem is combined with the reduction of state and parameter space. The inversion is based on a generalized datadriven optimization approach to construct the ROMs in [23] and enhanced with the Monte Carlo method to speed up the computations. The state projection $V \in \mathbb{R}^{N \times n}$ and parameter projection $P \in \mathbb{R}^{m \times m}$ are determined iteratively based on a greedy algorithm by maximizing the error between the high-fidelity original and the low-dimensional reduced model in the Bayesian setting.

Numerical experiments with the DCM model in [23] show the highly dimensional neuronal network system is efficiently inverted due to the short offline durations. In the offline phase, Monte Carlo-enhanced methods require more than an order of magnitude less offline time compared to the original and data misfit-enhanced methods. In the online phase the ROM has a speedup factor of about an order of magnitude more compared to the full-order inversion. The output error of the data misfit-enhanced method is close to that of the full-order method. The output errors of Monte Carlo decrease with increasing numbers of simulation but do not reach the output error of the full-order system. The source code is available in MATLAB [8]. 


\subsection{Dynamic mode decomposition}

DMD is a data-driven, equation-free ROM technique [20]. It was initially developed to reduce the high-dimensional dynamic data obtained from experiments and simulations in fluid mechanics into a small number of coupled spatio-temporal modes $[29,30]$. DMD was applied to explore spatio-temporal patterns in large-scale neuronal recordings in [3]. DMD can be interpreted as combination of discrete Fourier transform (DFT) in time and PCA [14] in space. Both PCA and independent component analysis [13] are static techniques, which perform poorly in reproducing dynamic data, such as recordings of neural activity.

The data are taken from electrocorticography (ECoG) recordings. Voltages from $n$ channels of an electrode array were sampled every $\Delta t$. These measurements are arranged at snapshot $k$ to the column vectors $\mathbf{x}_{k}$. The $m$ snapshots in time construct data matrices shifted in time with $\Delta t$,

$$
\mathbf{X}=\left[\begin{array}{cccc}
\mid & \mid & & \mid \\
\mathbf{x}_{1} & \mathbf{x}_{2} & \cdots & \mathbf{x}_{m-1} \\
\mid & \mid & & \mid
\end{array}\right], \quad \mathbf{X}^{\prime}=\left[\begin{array}{cccc}
\mid & \mid & & \mid \\
\mathbf{x}_{2} & \mathbf{x}_{3} & \cdots & \mathbf{x}_{m} \\
\mid & \mid & & \mid
\end{array}\right]
$$

These matrices are assumed to be related linearly in time,

$$
\mathbf{X}^{\prime}=\mathbf{A X}
$$

The DMD of the data matrix pair $\mathbf{X}$ and $\mathbf{X}^{\prime}$ is given by the eigendecomposition of $\mathbf{A}$ using the singular value decomposition of the data matrix $\mathbf{X}=U \Sigma V^{*}$ by computing the pseudo-inverse $\mathbf{A} \approx \mathbf{X}^{\prime} \mathbf{X}^{\dagger} \equiv \mathbf{X}^{\prime} \mathbf{V} \boldsymbol{\Sigma}^{-1} \mathbf{U}^{*}$. The spatio-temporal modes are computed by the exact DMD algorithm [32].

Because DMD does not contain explicit spatial relationships between neighboring measurements, traveling waves occurring in neuronal networks cannot be captured well with a few coherent modes. DMD was also used as a windowed technique with a temporal window size constrained by lower bound as for DFT. In contrast to the fluid dynamics where $n \gg m$, in neuroscience the electrode arrays have tens of channels $n$ in the recordings with $m$ number of snapshots in the windows data per second, so that $n<m$. The number of singular values $v$ of $\mathbf{X}$ are less than $n$ and $m-1$, which restricts the maximum number of DMD modes and eigenvalues to $n$. Because of this the dynamics can be captured over $m$ snapshots. The rank mismatch is resolved by appending to the snapshot measurements with $h-1$ time-shifted versions of the data matrices. The augmented data matrix $\mathbf{X}_{\text {aug }}$ is given as 


$$
\mathbf{X}_{\text {aug }}=\left[\begin{array}{cccc}
\mid & \mid & & \mid \\
\mathbf{x}_{1} & \mathbf{x}_{2} & \cdots & \mathbf{x}_{m-h} \\
\mid & \mid & & \mid \\
\mid & \mid & & \mid \\
\mathbf{x}_{2} & \mathbf{x}_{3} & \cdots & \mathbf{x}_{m-h-1} \\
\mid & \mid & & \mid \\
\mid & \mid & & \mid \\
\mathbf{x}_{h} & \mathbf{x}_{h+1} & \cdots & \mathbf{x}_{m-1} \\
\mid & \mid & & \mid
\end{array}\right]
$$

The augmented matrices $\mathbf{X}_{\text {aug }}$ and $\mathbf{X}_{\text {aug }}^{\prime}$ are Hankel matrices, which are constant along the skew diagonal, as in the eigenvalue realization algorithm [15]. The number of stacks $h$ is chosen such that $h n>2 m$. A measure to determined the optimal number of stacks $h$ is the approximation error

$$
E=\frac{\|\mathbf{X}-\hat{\mathbf{X}}\|_{F}}{\|\mathbf{X}\|_{F}},
$$

where $\|\cdot\|_{F}$ is the Frobenius norm. The approximation error $E$ is decreasing with increasing number of stacks $h$ and reaches a plateau, so that the DMD accuracy does not significantly increase.

DMD is applied in [3] as an automated approach to detect and analyze reliably spatial localization and frequencies of sleep spindle networks from human ECoG recordings. A MATLAB implementation is available at github.com/bwbrunton/dmd-neuro/.

\subsection{Reduced-order modeling of biophysical neuronal networks}

Recently ROMs for ODEs

$$
\dot{x}(t)=A(t) x(t)+f(x(t))+B u(t)
$$

were constructed using POD and the DEIM to investigate input-output behavior of the neuronal networks in the brain [22, 21], where $x(t)$ are state and $u(t)$ are input variables.

The model in [22] is based on the chemical reactions of molecules in synapses, that are the intercellular information transfer points of neurons. The signaling pathways in striatal synaptic plasticity are modeled in [19]. This model describes how certain molecules, which are a prerequisite for learning in the brain, act in synapses. The stoichiometric equations obey the law of mass action, which leads to a deterministic 
system of 44 ODEs of the form (7.27). The state $x(t)$ of the control system (7.27) is a collection of ions, molecules, and proteins that act in neuronal synapses. The linear part of (7.27) is sparse, the nonlinearities are quadratic. The time-dependent stimulus $u(t)$ consists of molecules that are important for neuronal excitability and plasticity, calcium and glutamate.

In [21], a nonlinear biophysical network model is considered, describing synchronized population bursting behavior of heterogeneous pyramidal neurons in the brain [27]. Neurons communicate by changing their membrane voltage to create action potentials (spikes), propagating from cell to cell. Spiking is the fundamental method of sensory information processing in the brain, and synchronized spiking is an emergent property of biological neuronal networks. The ODE system (7.27) in [21] consists of the states $x(t)$ as a collection of 50 neurons, each modeled with 10 ODEs, leading totally to a system of ODEs of dimension 500. Each cell is modeled with HH equations, where each cell has only two compartments (dendrites and soma) and these compartments have different ion channels. The state variables $x(t)$ include the voltages of somatic and dendritic compartments, the dendritic calcium concentration, and synaptic and ion channel gating variables, and the input $u(t)$ is an injected current. Additionally, the soma compartment voltages are coupled to dentritic compartments of randomly chosen cells. This networking of the output of cells as input to other cells is key for producing synchronized population behavior. The nonlinearities are $\mathrm{HH}$ type, i. e., exponential functions as well as cubic and quartic polynomials.

In [22], POD-DEIM was applied to a data-driven biological model of plasticity in the brain (7.27). The ROMs with POD-DEIM reduce significantly the simulation time and error between the original model and reduced-order solutions can be tuned by adjusting the number of POD and DEIM bases independently. When the ROMs are trained in a matching time interval of 10,000 seconds, accurate results are obtained. However, generalizing the reduced model to longer time intervals is challenging, which is characteristic for all nonlinear models.

In [21], the network model (7.27) is reduced with localized DEIM [24], discrete adaptive POD (DAPOD) [33, 34], and adaptive DEIM (ADEIM) [25]. DEIM and the variations are used here in combination with POD. ROMs require large numbers of POD and DEIM bases, to accurately simulate the input-output behavior in the ROMs. In this model, every cell is heterogeneous in parameters and there are also jump/reset conditions, which are factors that pose additional challenges to the ROMs. However, the ROMs in [21] were able to replicate the emergent synchronized population activity in the original network model. DAPOD and ADEIM perform best in preserving the spiking activity of the original network model. ADEIM is too slow and does not allow low enough dimensions to offset the computational costs of online adaptivity. DAPOD is able to find a lower-dimensional POD basis online than the other methods find offline, but the runtime is close to that of the original model. 


\section{Bibliography}

[1] D. Amsallem and J. Nordström, High-order accurate difference schemes for the Hodgkin-Huxley equations, J. Comput. Phys., 252 (2013), 573-590.

[2] D. Amsallem and J. Nordström, Energy stable model reduction of neurons by nonnegative discrete empirical interpolation, SIAM J. Sci. Comput., 38 (2) (2016), B297-B326.

[3] B. W. Brunton, L. A. Johnson, J. G. Ojemann, and J. N. Kutz, Extracting spatial-temporal coherent patterns in large-scale neural recordings using dynamic mode decomposition, $J$. Neurosci. Methods, 258 (2016), 1-15.

[4] L. Buesing, J. H. Macke, and M. Sahani, Spectral learning of linear dynamics from generalised-linear observations with application to neural population data, in F. Pereira, C. J.C. Burges, L. Bottou, and K. Q. Weinberger (eds.) Advances in Neural Information Processing Systems 25, pp. 1682-1690, Curran Associates, Inc., 2012.

[5] S. Chaturantabut and D. Sorensen, Nonlinear model reduction via discrete empirical interpolation, SIAM J. Sci. Comput., 32 (5) (2010), 2737-2764.

[6] J.P. Cunningham and Byron M. Yu, Dimensionality reduction for large-scale neural recordings, Nat. Neurosci., 17 (11) (2014), 1500-1509.

[7] K. J. Friston, L. Harrison, and W. Penny, Dynamic causal modelling, Neurolmage, 19 (4) (2003), 1273-1302.

[8] C. Himpe, OPTMOR - OPTimization-based Model Order Reduction (version 1.2), 2015.

[9] C. Himpe, Combined State and Parameter Reduction: For Nonlinear Systems with an Application in Neuroscience, Internationaler Fachverlag für Wissenschaft \& Praxis, 2016.

[10] C. Himpe and M. Ohlberger, Data-driven combined state and parameter reduction for inverse problems, Adv. Comput. Math., 41 (5) (2015), 1343-1364.

[11] M. Hines, Efficient computation of branched nerve equations, Int. J. Biomed. Comput., 15 (1) (1984), 69-76.

[12] A. L. Hodgkin and A. F. Huxley, A quantitative description of membrane current and its application to conduction and excitation in nerve, Bull. Math. Biol., 52 (1) (Jan 1990), 25-71.

[13] A. Hyvärinen and E. Oja, Independent component analysis: algorithms and applications, Neural Netw., 13 (4) (2000), 411-430.

[14] I. T. Jolliffe, Principal Component Analysis, Springer Series in Statistics, Springer-Verlag, New York, 2005.

[15] J. N. Juang and R. S. Pappa, An eigensystem realization algorithm for modal parameter identification and model reduction, J. Guid. Control Dyn., 8 (5) (1985), 620-627.

[16] A. R. Kellems, S. Chaturantabut, D. C. Sorensen, and S. J. Cox, Morphologically accurate reduced order modeling of spiking neurons, J. Comput. Neurosci., 28 (3) (2010), 477-494.

[17] A. R. Kellems, D. Roos, N. Xiao, and S. J. Cox, Low-dimensional, morphologically accurate models of subthreshold membrane potential, J. Comput. Neurosci., 27 (2) (2009), 161.

[18] C. Kemere, G. Santhanam, B. M. Yu, A. Afshar, S. I. Ryu, T. H. Meng, and K. V. Shenoy, Detecting neural-state transitions using hidden Markov models for motor cortical prostheses, J. Neurophysiol., 100 (4) (2008), 2441-2452.

[19] B. Kim, S. L. Hawes, F. Gillani, L. J. Wallace, and K. T. Blackwell, Signaling pathways involved in striatal synaptic plasticity are sensitive to temporal pattern and exhibit spatial specificity, PLoS Comput. Biol., 9 (3) (2013), e1002953.

[20] J. N. Kutz, S. L. Brunton, B. W. Brunton, and J. L. Proctor, Dynamic Mode Decomposition: Data-driven Modeling of Complex Systems, Society for Industrial and Applied Mathematics (SIAM), Philadelphia, PA, 2016. 
[21] M. Lehtimäki, L. Paunonen, and M.-L. Linne, Projection-based order reduction of a nonlinear biophysical neuronal network model, in 2019 IEEE 58th Conference on Decision and Control (CDC), pp. 1-6, 2019.

[22] M. Lehtimäki, L. Paunonen, S. Pohjolainen, and M.-L. Linne, Order reduction for a signaling pathway model of neuronal synaptic plasticity, IFAC-PapersOnLine, 50 (1) (2017), 7687-7692, 20th IFAC World Congress.

[23] C. Lieberman, K. Willcox, and O. Ghattas, Parameter and state model reduction for large-scale statistical inverse problems, SIAM J. Sci. Comput., 32 (5) (2010), 2523-2542.

[24] B. Peherstorfer, D. Butnaru, K. Willcox, and H.-J. Bungartz, Localized discrete empirical interpolation method, SIAM J. Sci. Comput., 36 (1) (2014), A168-A192.

[25] B. Peherstorfer and K. Willcox, Online adaptive model reduction for nonlinear systems via low-rank updates, SIAM J. Sci. Comput., 37 (4) (2015), A2123-A2150.

[26] B. Petreska, B. M. Yu, J. P. Cunningham, S. Gopal, S. I. Ryu, K. V. Shenoy, and M. Sahani, Dynamical segmentation of single trials from population neural data, in J. Shawe-Taylor, R. S. Zemel, P. L. Bartlett, F. Pereira, and K. Q. Weinberger (eds.), Advances in Neural Information Processing Systems 24, pp. 756-764, Curran Associates, Inc., 2011.

[27] P. F. Pinsky and J. Rinzel, Intrinsic and network rhythmogenesis in a reduced traub model for CA3 neurons, J. Comput. Neurosci., 1 (1) (1994), 39-60.

[28] S. T. Roweis and L. K. Saul, Nonlinear dimensionality reduction by locally linear embedding, Science, 290 (5500) (2000), 2323-2326.

[29] C. W. Rowley, I. Mezić, S. Bahheri, P. Schlatter, and D. S. Hennigson, Spectral analysis of nonlinear flows, J. Fluid Mech., 641 (2009), 115-127.

[30] P. J. Schmid, Dynamic mode decomposition of numerical and experimental data, J. Fluid Mech., 656 (2010), 5-28.

[31] J. B. Tenenbaum, V. de Silva, and J. C. Langford, A global geometric framework for nonlinear dimensionality reduction, Science, 290 (5500) (2000), 2319-2323.

[32] J. H. Tu, C. W. Rowley, D. M. Luchtenburg, S. L. Brunton, and J. N. Kutz, On dynamic mode decomposition: Theory and applications, J. Comput. Dyn., 1 (2) (2014), 391-421.

[33] M. Yang and A. Armaou, Dissipative distributed parameter systems on-line reduction and control using DEIM/APOD combination, in 2018 Annual American Control Conference (ACC), pp. 2557-2562, 2018.

[34] M. Yang and A. Armaou, Revisiting APOD accuracy for nonlinear control of transport reaction processes: A spatially discrete approach, Chem. Eng. Sci., 181 (2018), 146-158.

[35] B. M. Yu, J. P. Cunningham, G. Santhanam, S. I. Ryu, K. V. Shenoy, and M. Sahani, Gaussian-process factor analysis for low-dimensional single-trial analysis of neural population activity, J. Neurophysiol., 102 (1) (2009), 614-635. 